\title{
Targeting UDP-Glycosyltransferase, Glucosamine-6-Phosphate Synthase and Chitin Synthase by Using Bioactive 1,8 Cineole for "Aspergillosis" Fungal Disease Mutilating COVID-19 Patients: Insights from Molecular Docking, Pharmacokinetics and In-vitro Studies
}

\author{
Arun Dev Sharma ${ }^{1} \cdot$ Inderjeet Kaur $^{1}$
}

Received: 2 September 2021 / Accepted: 29 November 2021 / Published online: 7 January 2022

(c) The Tunisian Chemical Society and Springer Nature Switzerland AG 2021

\begin{abstract}
SARS-CoV-2 (COVID-19)-associated co-infections like "Aspergillosis", has recently baffled the world. Due to its key role in cell wall synthesis, in the present study UDP-glycosyltransferase, glucosamine-6-phosphate synthase and chitin synthase have been chosen as appropriate targets for molecular docking. The objective of the present study was molecular docking of eucalyptus essential oil component 1,8 cineole against cell wall enzymes followed by in vitro validation. For molecular docking, patch-dock web based online tool was used. Ligand-Protein 2D and 3D Interactions were also studied. Drug likeliness, toxicity profile and cancer cell line toxicity were also studied. Molecular docking results indicated that 1,8 cineole form hydrogen bonding and hydrophobic interactions with UDP-glycosyltransferase, glucosamine-6-phosphate synthase and chitin synthase enzymes. 1,8 cineole also depicted drug likeliness by showing compliance with the LIPINSKY rule, sufficient level of bioactivity and cancer cell line toxicity thus signifying its role as a potent anti-fungal drug.
\end{abstract}

Keywords Aspergillosis; COVID-19 · Eucalyptus oil · Herbal Drug

\section{Introduction}

The first case of pneumonia caused by SARS-CoV-2 (severe acute respiratory syndrome coronavirus 2) was first reported in China, Wuhan, in December 2019 [1]. Afterwards, this viral disease spread rapidly worldwide causing coronavirus disease (COVID-19) a pandemic. Since the dawn of COVID 19 pandemic, as of August 2020, researchers have documented COVID-19-associated serious co-infections in COVID-19 patients like: aspergillosis [1-3], invasive candidiasis [4], coccidioidomycosis [5], fusariosis [6], mucormycosis [7] and saccharomycosis [8]. Among all, "aspergillosis" contributed to a high mortality rate of up to $67 \%$ [9]. Aspergillosis" is a type of infection which is caused by common invasive fungus mold "Aspergillus", which exists in

Arun Dev Sharma

arundevsharma47@gmail.com

1 Post Graduate Department of Biotechnology, Lyallpur Khalsa College Jalandhar, Jalandhar, India outdoors and indoors. The rapid rise in fungal infections post 2nd wave of COVID-19 was attributed to the un-regulated use of steroids for COVID patients. It was observed that this fungus affects immune-compromised individuals like COVID-19 patients in recovering state and have diabetes or high un-controlled sugar levels [10]. Doctors observed that uncontrolled use of steroids for COVID patients reduced the body immunity and raised blood glucose level in diabetic and non-diabetic individuals due to poor physical activity which increased the rate of infection of fungus infection [11]. The symptoms of invasive "Aspergillosis" are: running nose, headache, stiffness, chest pain, cough, blood in cough, fever, reduced ability to smell and breathing problems [12].

Due to the rapid emergence of resistant strains of fungus and side effect of antifungal drugs, the synthesis and demand of novel drugs having less toxicity and more effectiveness is instantly required [13]. Hence bioactive molecules that pose properties to act as fungal cell wall-associated enzymes inhibitors have been advocated as key therapeutic drugs to treat fungal infections [14]. Fungal cell wall is a rigid mechanical barrier that plays an key role in protecting fungus 
against environmental stresses and other osmotic forces due to the presence of various structural components like chitin, glycosyl phosphatidyl inositol anchors (GPI), glucan and mannoproteins [15]. Therefore, these structural cell wallbased components represent excellent target sites to design antifungal drugs. Chitin synthase, UDP-glycosyltransferase and Glucosamine-6-phosphate synthase are key enzymes involved in cell wall construction. Earlier studies have paved the way that these components can serve as an excellent targets to design antifungal drugs as no such structures exist in human body [16]. We present here our viewpoint that bioactive molecule eucalyptol also known as 1,8 cineole has the potential to treat Aspergillosis infection by targeting fungal enzymes such as Chitin synthase, UDP-glycosyltransferase and Glucosamine-6-phosphate synthase. Chitin synthase is involved in the process of chitin biosynthesis [16]. UDPglycosyltransferase is a key enzyme involved in the first step in glycosyl phosphatidyl inositol GPI biosynthesis. GPI is a potential molecule needed for anchoring proteins to the cell membrane thus involved in the integrity of the fungal cell wall [17]. Glucosamine-6-phosphate synthase is involved in the syntheses of $\mathrm{N}$-acetylglucosamine which is an essential building block for fungal cell wall chitin [18].

Eucalyptus essential oil from eucalyptus species encompasses a number of bioactives. Among all, our previous GCFID phytochemical based studies revealed that 1,8-cineole (eucalyptol) is a main bioactive of eucalyptus oil in Eucalyptus globules [19]. Due to the complex nature of essential oil, their anti-fungal mechanism of action is still not completely understood [20]. Previously antifungal potential of leaf hot water extracts against dermatophytes, filamentous and Candida albicans has been cited [19-21]. This study postulated that due to the richness of 1,8 cineole, essential oil from Eucalyptus globules plants have the potential to inhibit "Aspergillosis". Hence as an objective this study was designed to study molecular docking of 1,8 cineole against Chitin synthase, UDP-glycosyltransferase and Glucosamine6-phosphate synthase and wet-lab validation. The present study outcomes would offer new prospects to identify the key antifungal drugs during COVID19 medications.

\section{Materials and Methods}

\subsection{Ligand Modelling}

1,8 cineole was ligand for Chitin synthase, UDP-glycosyltransferase and Glucosamine-6-phosphate synthase structures. SMILES of 1,8 cineole $(\mathrm{CC} 1(\mathrm{C} 2 \mathrm{CCC}(\mathrm{O} 1)(\mathrm{CC} 2) \mathrm{C})$ C) were retrieved with PubChem CID 2758 from the NCBIPubchem database. UCSF-chimera build structure option was used to build its 3D structure of 1,8 cineole which was then saved a pdb file.

\subsection{Protein Receptor Preparation and Molecular Docking}

X-ray crystal structures of Chitin synthase, UDP-glycosyltransferase and Glucosamine-6-phosphate synthase with PDB IDs: 4gf8, 5u6m and $1 \mathrm{jxa}$, respectively were retrieved from PDB web site (https://www.rcsb.org/). The target enzymes were cleaned from co-crystallized ligand, selected water molecules and cofactors, prepared energy minimized before docking study. Before the docking studies, the protein structure was first prepared using the dock prep set up in chimera software. The dock preparation is an optimization part that corrects atomic and bond length, structure, and charges anomalies. Original inhibitors and water molecules were detached from the Chitin synthase, UDP-glycosyltransferase and Glucosamine-6-phosphate synthase structures and any missing hydrogen atoms were added. PatchDock tool was used for docking study of the 1,8 cineole over Chitin synthase, UDP-glycosyltransferase and Glucosamine-6-phosphate synthase enzyme (https:// bioinfo3d.cs.tau.ac.il/PatchDock/). For this both ligand ( 1,8 cineole) and receptors molecules in.pdb file formats were uploaded to the PatchDock server and the job was executed. The best generated docked structure was downloaded and saved as.pdb file. Biovia Discovery Studio Visualizer 2020, and Plip tool (https://plip-tool.biotec. tu-dresden.de/plip-web/plip/index) were used to study docked complexes and their 2D and 3D interactions. For this, docked complex in pdb format was uploaded and job was executed with using default parameters.

\subsection{Drug-Likeness and Toxicity}

To calculate drug-likeness of 1,8 cineole, SMILES $(\mathrm{CC} 1(\mathrm{C} 2 \mathrm{CCC}(\mathrm{O} 1)(\mathrm{CC} 2) \mathrm{C}) \mathrm{C})$ were used. Various physiochemical properties, drug- likeness and pharmacokinetics studies and ADMET (Absorption, Metabolism, Toxicity and Excretion) study of 1,8 cineole were conducted using SWISSADME (http://www.swissadme.ch/ http://lmmd. ecust.edu.cn/admetsar1/predict/). For this SMILES of 1,8 cineole were submitted to SWISSADME server and job was executed. The toxicity profile was studied by using the ProTox-II webserver (http://tox.charite.de/protox_II). It calculates prediction based on different levels of toxicity such as organ toxicity (hepatotoxicity), oral toxicity, toxicological endpoints (such as cytotoxicity, carcinotoxicity, mutagenicity and immunotoxicity). Web based molinspiration tool was used to evaluate the bioactivity potential of 1,8 cineole (https://www.molinspiration.com/cgi-bin/properties). For toxicity and bioactivity calculation, SMILES of 1,8 cineole were uploaded to respective servers and jobs were executed. 


\subsection{Active Sites Prediction in 3D Modeled Receptor}

CASTp (The Computed Atlas of Surface Topography of proteins) web tool was used to predict active sites residues in the Chitin synthase, UDP-glycosyltransferase and Glucosamine-6-phosphate synthase proteins. CASTp is an online tool used in the identification and dimension of cavities on 3D protein structures. The default value of 1.4 Angstroms was used as probe radius. For this molecular structures of Chitin synthase, UDP-glycosyltransferase and Glucosamine-6-phosphate synthase proteins in pdb format were uploaded to CASTp server and the job was executed and output was analyzed.

\subsection{Cell Line Toxicity Prediction}

In-silico Cancer cell line toxicity analysis of 1,8 cineole was evaluated by using CLC-Pred web- based tool (http:// way2drug.com/Cell-line/). For this, input SMILES of 1,8 cineole were submitted to server, the job was executed using the default parameters and output results were analyzed.

\subsection{In-vitro Antifungal Activity of Eucalyptus Oil}

Two fungal cultures; Aspergillus niger (MTCC 152) and Aspergillus oryzae (MTCC 153) were procured from Institute of Microbial Technology, Chandigarh (India). Strains were maintained on PDA media (Hi-media). Leaves of Eucalyptus globules were collected from campus fields and plots. Eucalyptus oil from leaves of was extracted by using Steam-distillation method as described in Sharma et al. [19]. Oil obtained was stored in dark bottles at $4{ }^{\circ} \mathrm{C}$ till further use. To analyze the antifungal effect of eucalyptus oil on fungal strains, specific concentrations $(5-30 \mu \mathrm{l} / \mathrm{ml})$ of eucalyptus oil were mixed with molten PDA medium followed by manual rotation in Erlenmeyer flask to disperse the oil equally into the medium. $20 \mathrm{ml}$ of mixed medium was poured into sterile petri plates $(9 \mathrm{~cm}$ in diameter). Plates were allowed to solidify at room temperature $\left(\sim 35^{\circ} \mathrm{C}\right)$ for $2 \mathrm{~h}$. Mycelial fungal agar discs $(6 \mathrm{~mm})$ were taken from the margin of plates having active fungal growth from the 10-day-old pure fungal cultures with the help of sterile cork borer and aseptically inoculated at the middle of the petri plates. Streptomycin $(30 \mathrm{mg})$ was used a positive control. Control plates were inoculated in the same manner except for essential oil. All plates in triplicate were incubated at $28{ }^{\circ} \mathrm{C}$. After 5 days Inhibition of mycelial growth inhibition was determined by using formula $(\%)=\mathrm{DC}-\mathrm{DT} / \mathrm{DC} \times 100, \mathrm{DC}$ and DT: diameter of control and test colony.

\section{Results and Discussion}

Earlier observations cited that COVID patients who are in immune-compromised condition or having uncontrolled diabetes are infected by fungus disease also known as "Aspergillosis" [1-9]. Bio active compounds with antifungal activities disable fungus strains by targeting key components of fungal metabolism like cell-walls enzymes. Studies revealed that drugs targeting fungal cell wall components like chitin synthase, UDP-glycosyltransferase and Glucosamine-6-phosphate synthase can be promising antifungal therapeutic agents as no such structure exits in humans [15-18]. Since cell wall-based enzymes are pivotal for fungus survival, it is essential to exploits them as a key target of anti-fungal agent. Hence, chitin synthase, UDP-glycosyltransferase and Glucosamine-6-phosphate synthase may offer a new active fungicidal approach to treat "Aspergillosis". In view of this, the molecular docking study was carried out to examine the binding interactions of 1,8 cineole with chitin synthase, UDP-glycosyltransferase and Glucosamine-6-phosphate synthase.

\subsection{Molecular Docking}

Among all, structure-based drug design (SBDD) is most commonly used, which is based on 3-D structure [22]. In SBDD, Molecular docking is a key technique that can be applied in designing drug- making process. In-silico docking has facilitated researchers to monitor conformations and affinities of a collection of bio-actives against receptors [23]. This study investigated the docking of 1,8 cineole bioactive molecule from eucalyptus oil as key fungal inhibitor candidate against chitin synthase, UDP-glycosyltransferase and Glucosamine-6-phosphate synthase enzymes. In-silico docking results based on docking score and area are demonstrated in Table 1. Among all enzymes, it was found that Glucosamine-6-phosphate synthase depicted strong docking with 1,8 cineole as evident from dock score of 3670 . Docking score for chitin synthase and UDP-glycosyltransferase was 3278 and 3232, respectively. Docking pose and molecular interactions of 1,8 cineole with chitin synthase, UDP-glycosyltransferase and Glucosamine-6-phosphate synthase are shown in Fig. 1. It was observed that 1,8 cineole successfully docked in the active sites of with chitin synthase, UDP-glycosyltransferase and Glucosamine-6-phosphate synthase. Chitin synthase is a membrane bound enzyme complex having three domains: an $\mathrm{N}$-terminal domain, a catalytic domain and a C-terminal trans-membrane domain. From in-silico analysis it was found that 1,8 cineole exhibited its interaction with catalytic domain involved in chitin chain elongation between 
Table 1 Molecular docking of fungal receptors

\begin{tabular}{|c|c|c|c|c|c|c|c|}
\hline \multirow[t]{2}{*}{ Fungal Receptor } & \multicolumn{4}{|c|}{ Dock score } & \multicolumn{2}{|c|}{ Interacting residues within $4 \AA$ radius } & \multirow[t]{2}{*}{ Hydrogen bonds } \\
\hline & Score & Area & ACE & Transformation & Water bridge & Hydrophobic interactions & \\
\hline Chitin synthase & 3278 & 344.70 & -55.88 & $\begin{array}{l}0.871 .422 .56-4.58 \\
\quad-29.6313 .46\end{array}$ & GLN412 & PRO48, 131, TRP306 & - \\
\hline UDP-glucosyltransferase & 3232 & 351.70 & -66.24 & $\begin{array}{l}0.240 .582 .7474 .9981 .74 \\
28.34\end{array}$ & - & LYS334, VAL356 & - \\
\hline $\begin{array}{l}\text { Glucosamine-6-phosphate } \\
\text { synthase }\end{array}$ & 3670 & 387.30 & -106.05 & $\begin{array}{c}-1.581 .130 .683 .05 \\
32.0168 .97\end{array}$ & THR302 & HIS504, & SER401 \\
\hline
\end{tabular}

Fig. 1 Molecular docking of 1,8 cineole with fungal cell wall receptors

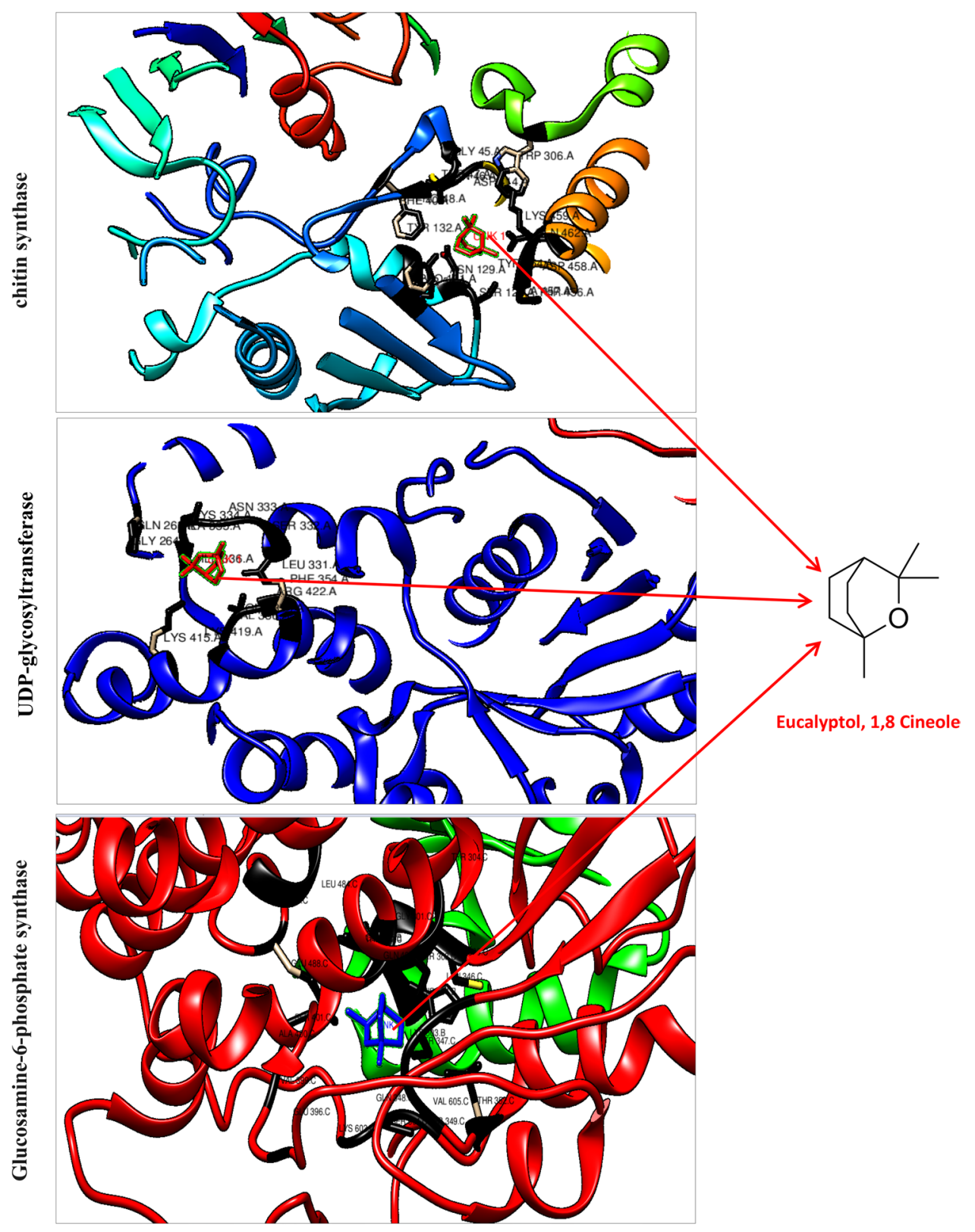


$\mathrm{N}$ and $\mathrm{C}$ terminal domains [24]. Glucosamine-6-phosphate synthase posse $\mathrm{N}$-terminal and $\mathrm{C}$-terminal ones, catalyzing glutamine hydrolysis and sugar-phosphate isomerization $[25,26]$. Computational analysis revealed that 1,8 cineole successfully docked with $\mathrm{C}$ terminal domain involved in sugar-phosphate isomerization. UDP-glycosyltransferase enzyme has two-domain structures: N-domain for the binding site of the aglycone substrate and $\mathrm{C}$ - terminal likely site of UDP-glucose binding [27]. The docking analysis exhibited interaction of 1,8 cineole with $\mathrm{C}$-terminal UDP-glucose binding domain. These results are in agreement with those of $[28,29]$ as they stated that molecular docking analyses were performed to clarify the antifungal effectiveness of the most active compounds of essential oil from Trachyaspermum ammi, Thymus vulgaris and Boswellia carteri against fungal enzymes. This study indicates that eucalyptus essential oil may be considered as the most important sources of antifungal compounds.

During docking drug molecule either forms hydrophobic interactions or hydrogen bonding with in the active site residues of receptor that determines affinity of ligand with receptor. So molecular interactions of 1,8 cineole with chitin synthase, UDP-glycosyltransferase and Glucosamine-6-phosphate synthase were further evaluated. It was observed that the interaction of 1,8 cineole in active sites of chitin synthase, UDP-glycosyltransferase and Glucosamine-6-phosphate synthase was mediated by both hydrophobic and hydrogen bond interactions. With Glucosamine-6-phosphate synthase hydrophobic interactions were observed via HIS 504 (Fig. 2). Hydrogen bond interactions of 1,8 cineole with Glucosamine6-phosphate synthase were also observed via SER 401. For chitin synthase, hydrophobic interactions were observed via PRO48, 131, TRP306. With UDP-glucosyltransferase, hydrophobic interactions were observed via LYS334 and VAL356. No Hydrogen bond interactions were observed with chitin synthase, UDP-glycosyltransferase. Greater the hydrogen bonds between the enzyme and ligand determines the strength of binding [30]. In view of this, due to having hydrogen bond interaction, 1,8 cineole depicted strong binding with Glucosamine-6-phosphate synthase as compared to other enzymes which was also evident from its docking score. Active site prediction by CAST-P server indicated interacting residues in the major cavity of chitin synthase, UDP-glycosyltransferase and Glucosamine-6-phosphate synthase enzymes (Table 2). With CASTp, a major pocket was identified with Area (SA) of 939 and Volume (SA) of 3369 in chitin synthase. While Area (SA) of 1648 and Volume (SA) of 1198 were observed for Glucosamine-6-phosphate synthase. Since 1,8 cineole poses high affinity towards chitin synthase, UDP-glycosyltransferase and Glucosamine-6-phosphate enzymes so it was postulated that chitin synthase, UDP-glycosyltransferase and Glucosamine-6-phosphate proteins becomes closed upon binding with 1,8 cineole that in turn induces a conformational change in

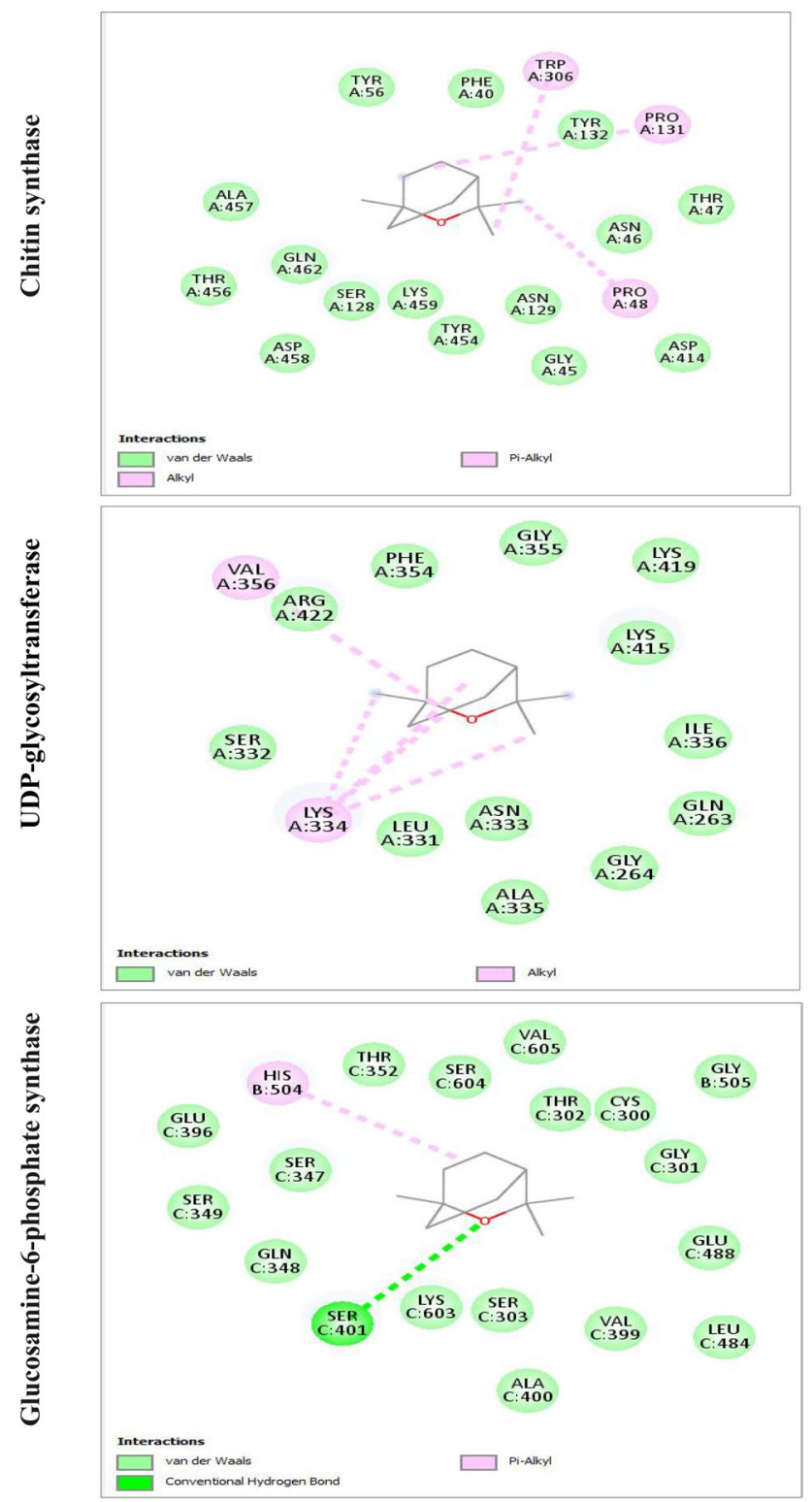

Fig. 2 2D interactions of 1,8 cineole with protein receptors

chitin synthase, UDP-glycosyltransferase and Glucosamine6-phosphate proteins and stop further execution of catalysis of fungal cell wall synthesis hence down-regulate the infectivity of fungus into host cell. The present study results were in consonance with the earlier in-silico findings suggesting that polypharmacological agents via cell wall inhibition can act as a therapeutic for the management of Aspergillosis among COVID-19 patients [31, 32].

\subsection{PASS analysis, In-silico Bioactivity, Cell Toxicity and ADMET Properties}

For therapeutic use of drugs in living organisms, ADMET properties (absorption, distribution, metabolism, excretion 
Table 2 Protein target structure, native ligand and active site amino acids

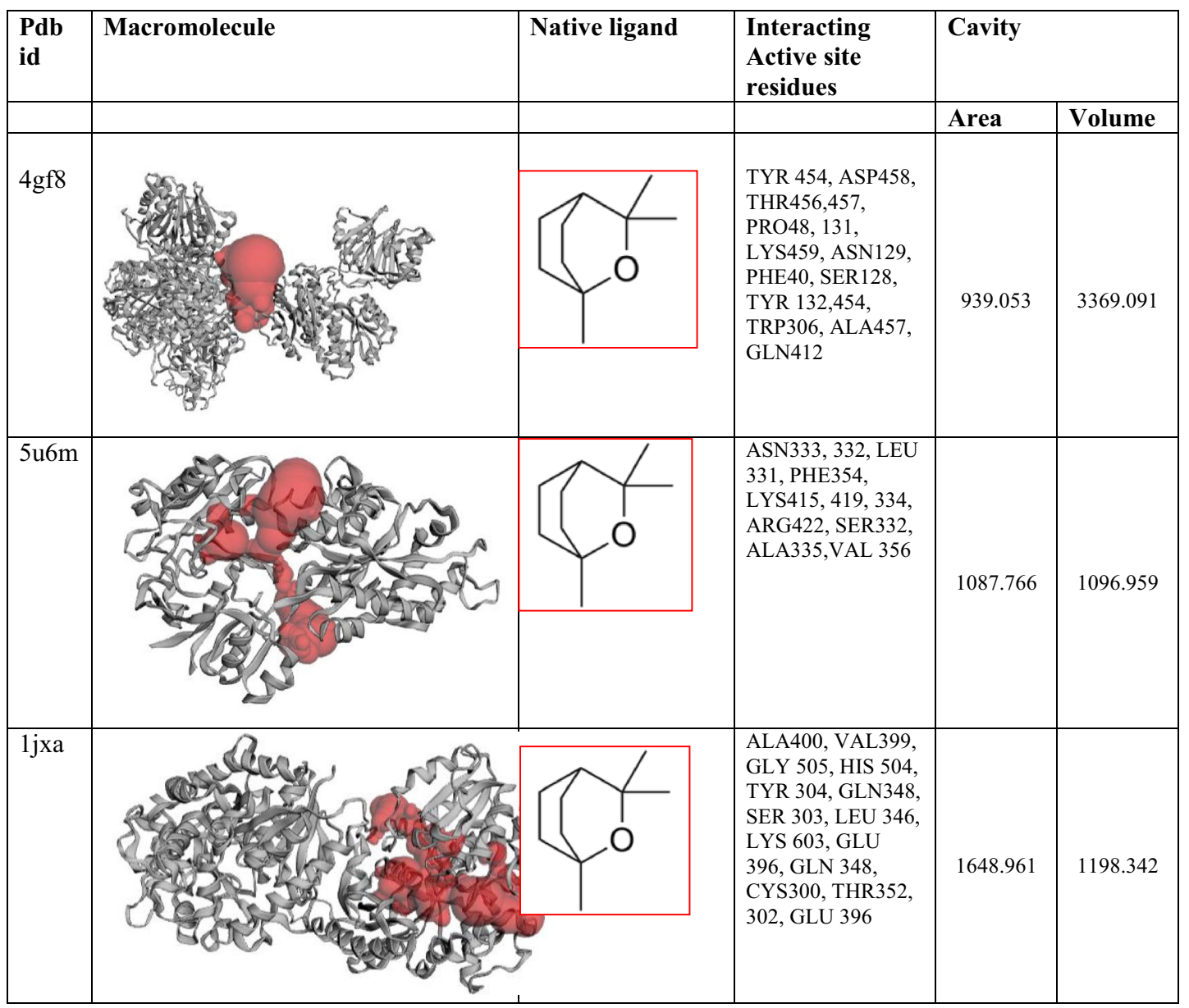

and toxicity) are very imperative for the success of any drug [29]. To find out drug likeliness, the Lipinski rule of 5 (RO5) is generally used. It is based on some molecular parameters like TPSA (polar surface area), mlog $\mathrm{P}$ (partition coefficient), number of hydrogen bond donors, molecular weight and number of hydrogen bond acceptors. According to this rule for drug-like properties ligands should have $\log \mathrm{P} \leq 5$, number of $\mathrm{H}$-bond acceptors $\leq 10$, and $\mathrm{H}$-bond donors $\leq 5$ and no more than 1 violation. As shown in Table 3, 1,8 cineole has shown good agreement with the given criteria. Hence, it was postulated that bioactive compound 1,8 cineole could be considered an oral drug [29]. In-silico absorption of 1,8 cineole was $100 \%$. It was observed that 1,8 cineole was a low molecular weight ligand. It was cited that low MWT compounds are easily diffused and transported across the biological membranes as compared to high MWT compounds [30]. The $\log P_{\mathrm{o} / \mathrm{w}}$ value was also in acceptable range. In rational drug design and pharmacokinetic analysis, $\log P_{\mathrm{o} / \mathrm{w}}$ is a key parameter to asses the lipophilicity of any drug and its distribution in the body after absorption [29, 30]. The surface view depicting molecular lipophilicity potential (MLP) is also shown in Fig. 3. MLP is convenient property to rationalize numerous molecular ADME characteristics (for example: plasma-protein binding or membrane penetration). Analysis of 3D distribution of hydrophobicity on molecular surface is predominantly helpful when explaining differences are observed in ADME properties of molecules with the same $\log P_{\mathrm{o} / \mathrm{w}}[28-31]$. The topological polar surface area (TPSA) value was $9.23 \AA$ squared. Topological polar surface area is a key predictor of drug transport properties such as nice permeability, bioavailability and intestinal absorption [28-31]. GI (Gastrointestinal tract absorption) of 1,8 cineole was high (Table 3 ). In order to exert a toxic effect, drug molecules have to be absorbed from intestinal tract in the body. Further, 1,8 cineole was non substrate to efflux transporters such as P-glycoprotein (P-gp). In the gut, P-glycoprotein pumps drugs back into the lumen, decreasing their absorption [33]. 1,8 cineole bioactive compound elaborated non-inhibitory potential against CYP450 series of enzymes, involved in liver detoxification in body [34, 35]. These observations indicated that 1,8 cineole can easily interact with target receptors and can be further taken in the evaluation of biological activity score. 
Table 3 Physicochemical Properties and

Pharmacokinetics properties of

1,8 cineole

\begin{tabular}{|c|c|}
\hline \multicolumn{2}{|l|}{ Physicochemical properties } \\
\hline Formula & $\mathrm{C} 10 \mathrm{H} 18 \mathrm{O}$ \\
\hline Molecular weight & $154.25 \mathrm{~g} / \mathrm{mol}$ \\
\hline Num. heavy atoms & 11 \\
\hline Num. arom. heavy atoms & 0 \\
\hline Fraction Csp3 & 1.00 \\
\hline Num. rotatable bonds & 0 \\
\hline Num. H-bond acceptors & 1 \\
\hline Num. H-bond donors & 0 \\
\hline Molar Refractivity & 47.12 \\
\hline TPSA 2 & $9.23 \AA^{2}$ \\
\hline \multicolumn{2}{|l|}{ Lipophilicity } \\
\hline $\log P_{\mathrm{o} / \mathrm{w}}(\mathrm{iLOGP})$ & 2.58 \\
\hline $\log P_{\mathrm{o} / \mathrm{w}}(\mathrm{XLOGP3})$ & 2.74 \\
\hline $\log P_{\mathrm{o} / \mathrm{w}}(\mathrm{WLOGP})$ & 2.74 \\
\hline $\log P_{\mathrm{o} / \mathrm{w}}(\mathrm{MLOGP})$ & 2.45 \\
\hline $\log P_{\mathrm{o} / \mathrm{w}}($ SILICOS-IT) $?$ & 2.86 \\
\hline Consensus $\log P_{\mathrm{o} / \mathrm{w}}(2$ & 2.67 \\
\hline \multicolumn{2}{|l|}{ Pharmacokinetics } \\
\hline GI absorption (?) & High \\
\hline BBB permeant 2 & Yes \\
\hline P-gp substrate 2 & No \\
\hline CYP1A2 inhibitor 2 & No \\
\hline CYP2C19 inhibitor 2 & No \\
\hline CYP2C9 inhibitor 2 & No \\
\hline CYP2D6 inhibitor 2 & No \\
\hline CYP3A4 inhibitor 2 & No \\
\hline $\log K_{\mathrm{p}}$ (skin permeation) & $-5.30 \mathrm{~cm} / \mathrm{s}$ \\
\hline \multicolumn{2}{|l|}{ Druglikeness } \\
\hline Lipinski (2) & Yes; 0 violation \\
\hline Ghose 2 & No; 1 violation: $\mathrm{MW}<160$ \\
\hline Veber $?$ & Yes \\
\hline Egan 2 & Yes \\
\hline Muegge (2) & $\begin{array}{l}\text { No; } 2 \text { violations: } \mathrm{MW}<200 \text {, } \\
\text { Heteroatoms }<2\end{array}$ \\
\hline Bioavailability Score (2) & 0.55 \\
\hline \multicolumn{2}{|l|}{ Medicinal chemistry } \\
\hline PAINS ? & 0 alert \\
\hline Brenk 3 & 0 alert \\
\hline Leadlikeness (2) & No; 1 violation: $\mathrm{MW}<250$ \\
\hline Synthetic accessibility 2 & 3.65 \\
\hline
\end{tabular}



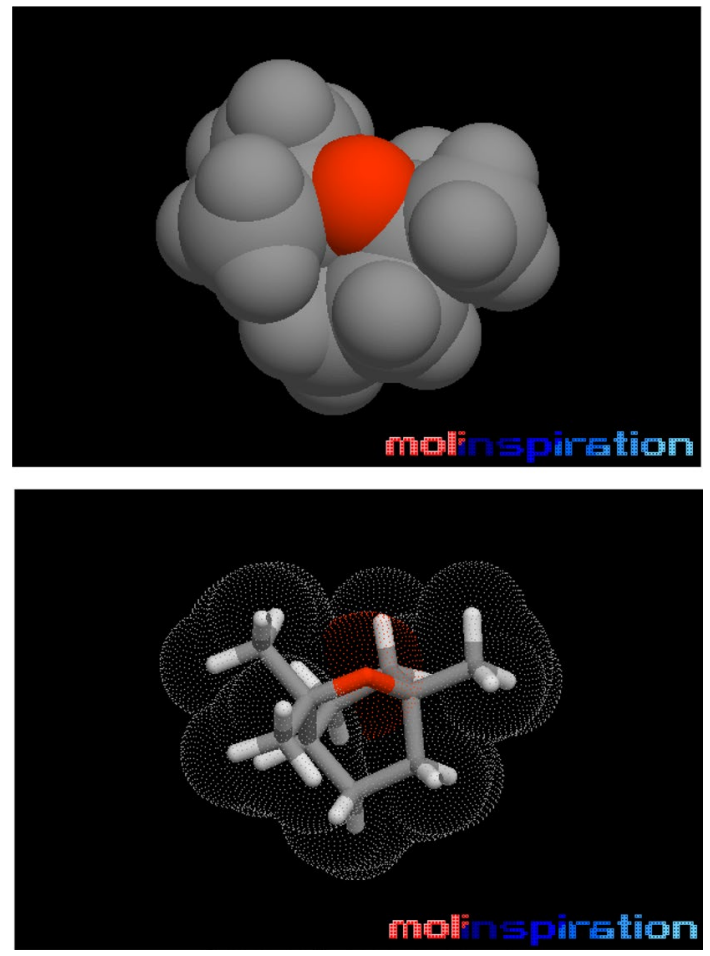

Fig. 3 Molecular lipophilicity potential (MLP)/polar surface area (PSA) views of 1,8 cineole. Hydrophobic areas: encoded by violet; Hydrophilic areas: red

Table 4 Bioactivity score of 1,8 cineole

\begin{tabular}{ll}
\hline Bioactivity & Score \\
\hline GPCR ligand & -0.93 \\
Ion channel modulator & 0.01 \\
Kinase inhibitor & -1.60 \\
Nuclear receptor ligand & -1.07 \\
Protease inhibitor & -0.90 \\
Enzyme inhibitor & -0.1 \\
\hline
\end{tabular}

Biological activity is a key parameter which describes the effect of a drug in living systems. In living systems, ligands have to be bound to biological targets which are also known as drug targets [36]. Drug targets mostly include common proteins such as enzymes, receptors and ion channels. Bioactivity score was calculated with online Molinspiration software based on following parameters such as binding to GPCR ligand, Ion channel modulator,Kinase inhibitor, Nuclear receptor ligand, Protease inhibitor and Enzyme inhibitor. This score as per rule is calculated in three different ranges: score $>0$, drug is active, if it is between -5.0 and 0 , drug is judiciously active and if score $<$ than -5.0 , drug is quiet. For 1,8 cineole, bioactivity score for Ion channel modulator was 0.01 whereas for GPCR ligand, Kinase inhibitor, Nuclear receptor ligand, Protease inhibitor and Enzyme inhibitor score was in the range of -5.0 and 0 ,
(Table 4). All these observations indicated that 1,8 cineole possess such properties as are required for the bio active molecules to act as potential drugs. Similar observations have been reported by researchers working on different drug formulations [36, 37]. The bioactivity score deliver the evidence about the binding cascade of the 1,8 cineole that is used for the improvement of a new functional drug with increased binding selectivity profile and less undesirable effects $[35,36]$

For pharmaceutical industries, proper risk assent of a chemical drug is a prerequisite to assess the safety profile of a therapeutic drug [38]. In this regard, in silico toxicity is a key platform to evaluate toxicity prediction of drugs that could be detrimental to humans, animals, and environments [39]. Thus toxicity profile of 1,8 cineole was evaluated and toxicity profile revealed that 1,8 cineole bioactive molecule was mostly non toxic to organs as inactive prediction was observed like hepatotoxicity (Table 5). Drug-induced hepatotoxicity is the major reason for liver damage and the main reason for the un-success of major drugs in the market [40]. Further, 1,8 cineole was non-carcinogenic and non-mutagenic. Mutagenic nature of biomolecules is harmful to cell and is the main reason behind certain diseases, e.g. cancer [41]. Further 1,8 cineole showed inactiveness towards targets-based on biological pathways like Nuclear receptor signaling pathways and Stress response pathways. All these targets like aryl hydrogen receptor (AhR), androgen receptor (AR), androgen receptor ligand binding domain (ARLBD), 2/antioxidant responsive element (ARE), heat shock factor response element (HSE), mitochondrial membrane potential (MMP) are important components of biological system inside human body [42]. Toxicity radar chart is also shown in Fig. 4, that quickly exemplifies the assurance of positive toxicity outcomes compared to the average of its class. Further, 1,8 cineole depicted cell line toxicity to tumor cell lines (Table 6). As per Way2Drug server prediction, it was found that values of $\mathrm{Pa}>$ values of $\mathrm{Pi}$, depicted that 1,8 cineole compound was belonged to the sub-class of active compounds i.e. it resembles the structures of molecules, which are the most typical in a sub-set of "actives" in PASS training set.

\subsection{In vitro Antifungal Activity of Eucalyptus Oil}

In order to validate the in-silico findings, a wet-lab experiment was designed to evaluate the antifungal potential of eucalyptus oil against two fungal strains: Aspergillus niger and Aspergillus oryzae. In both fungal strains, complete mycelial growth inhibition was observed at essential oil concentration of 30 $\mu \mathrm{L} / \mathrm{mL}$ (Fig. 5, Table 7). Eucalyptus essential oil depicted significant antifungal activity (mycelial growth inhibition was 40-50\%) against Aspergillus oryzae and Aspergillus niger at a concentration of $5 \mu \mathrm{l} / \mathrm{ml}$ after 5 days of incubation. 
Table 5 Toxicity model report of 1,8 cineole

\begin{tabular}{|c|c|c|c|}
\hline Classification & Target & Prediction & Probability \\
\hline Organ toxicity & Hepatotoxicity & Inactive & 0.86 \\
\hline Toxicity end points & Carcinogenicity & Inactive & 0.68 \\
\hline Toxicity end points & Immunotoxicity & Inactive & 0.99 \\
\hline Toxicity end points & Mutagenicity & Inactive & 0.96 \\
\hline Toxicity end points & Cytotoxicity & Inactive & 0.75 \\
\hline $\begin{array}{l}\text { Tox21-Nuclear } \\
\text { receptor signalling } \\
\text { pathways }\end{array}$ & Aryl hydrocarbon Receptor (AhR) & Inactive & 0.98 \\
\hline $\begin{array}{l}\text { Tox21-Nuclear } \\
\text { receptor signalling } \\
\text { pathways }\end{array}$ & Androgen Receptor (AR) & Inactive & 0.99 \\
\hline $\begin{array}{l}\text { Tox21-Nuclear } \\
\text { receptor signalling } \\
\text { pathways }\end{array}$ & $\begin{array}{l}\text { Androgen Receptor Ligand } \\
\text { Binding Domain (AR-LBD) }\end{array}$ & Inactive & 1.0 \\
\hline $\begin{array}{l}\text { Tox21-Nuclear } \\
\text { receptor signalling } \\
\text { pathways }\end{array}$ & Aromatase & Inactive & 0.98 \\
\hline $\begin{array}{l}\text { Tox21-Nuclear } \\
\text { receptor signalling } \\
\text { pathways }\end{array}$ & Estrogen Receptor Alpha (ER) & Inactive & 0.96 \\
\hline $\begin{array}{l}\text { Tox21-Nuclear } \\
\text { receptor signalling } \\
\text { pathways }\end{array}$ & $\begin{array}{l}\text { Estrogen Receptor Ligand Binding } \\
\text { Domain (ER-LBD) }\end{array}$ & Inactive & 0.97 \\
\hline $\begin{array}{l}\text { Tox21-Nuclear } \\
\text { receptor signalling } \\
\text { pathways }\end{array}$ & $\begin{array}{l}\text { Peroxisome Proliferator Activated } \\
\text { Receptor Gamma (PPAR- } \\
\text { Gamma) }\end{array}$ & Inactive & 0.99 \\
\hline $\begin{array}{l}\text { Tox21-Stress } \\
\text { response pathways }\end{array}$ & $\begin{array}{l}\text { Nuclear factor (erythroid-derived } \\
\text { 2)-like 2/antioxidant responsive } \\
\text { element (nrf2/ARE) }\end{array}$ & Inactive & 0.99 \\
\hline $\begin{array}{l}\text { Tox } 21-\text { Stress } \\
\text { response pathways }\end{array}$ & $\begin{array}{l}\text { Heat shock factor response } \\
\text { element (HSE) }\end{array}$ & Inactive & 0.99 \\
\hline $\begin{array}{l}\text { Tox21-Stress } \\
\text { response pathways }\end{array}$ & $\begin{array}{l}\text { Mitochondrial Membrane Potential } \\
\text { (MMP) }\end{array}$ & Inactive & 0.89 \\
\hline $\begin{array}{l}\text { Tox21-Stress } \\
\text { response pathways }\end{array}$ & $\begin{array}{l}\text { Phosphoprotein (Tumor } \\
\text { Supressor) p53 }\end{array}$ & Inactive & 0.99 \\
\hline $\begin{array}{l}\text { Tox21-Stress } \\
\text { response pathways }\end{array}$ & $\begin{array}{l}\text { ATPase family AAA domain- } \\
\text { containing protein } 5 \text { (ATAD5) }\end{array}$ & Inactive & 0.99 \\
\hline
\end{tabular}

Probability $(\mathrm{P})$ indicates toxicity confidence score of the 1,8 cineole. score $>1$ : toxic (active)
Full mycelial growth inhibition (100\%) was observed at a concentration of $30 \mu \mathrm{l} / \mathrm{ml}$. A substantial antifungal activity was also observed with positive control streptomycin which was reported to be a fungistatic or fungicidal [43]. The strong antifungal activity of eucalyptus essential oil may be due to the richness of eucalyptol. These observations were in consonance with earlier studies showing the antifungal effect of methanolic extracts of eucalyptus essential oil against Alternaria alternate, a pathogenic fungus causing leaf spot infection in plants [44]. Antifungal effect of eucalyptus essential oil has been reported against other pathogenic fungi strains like: Penicillium digitatum, Fusarium solani, Colletotrichum gloeosporioides, Pythium ultimum, Rhizoctonia solani, Bipolaris sorokiniana, Fusarium graminearum, and Fusarium sporotrichioides $[45,46]$. 


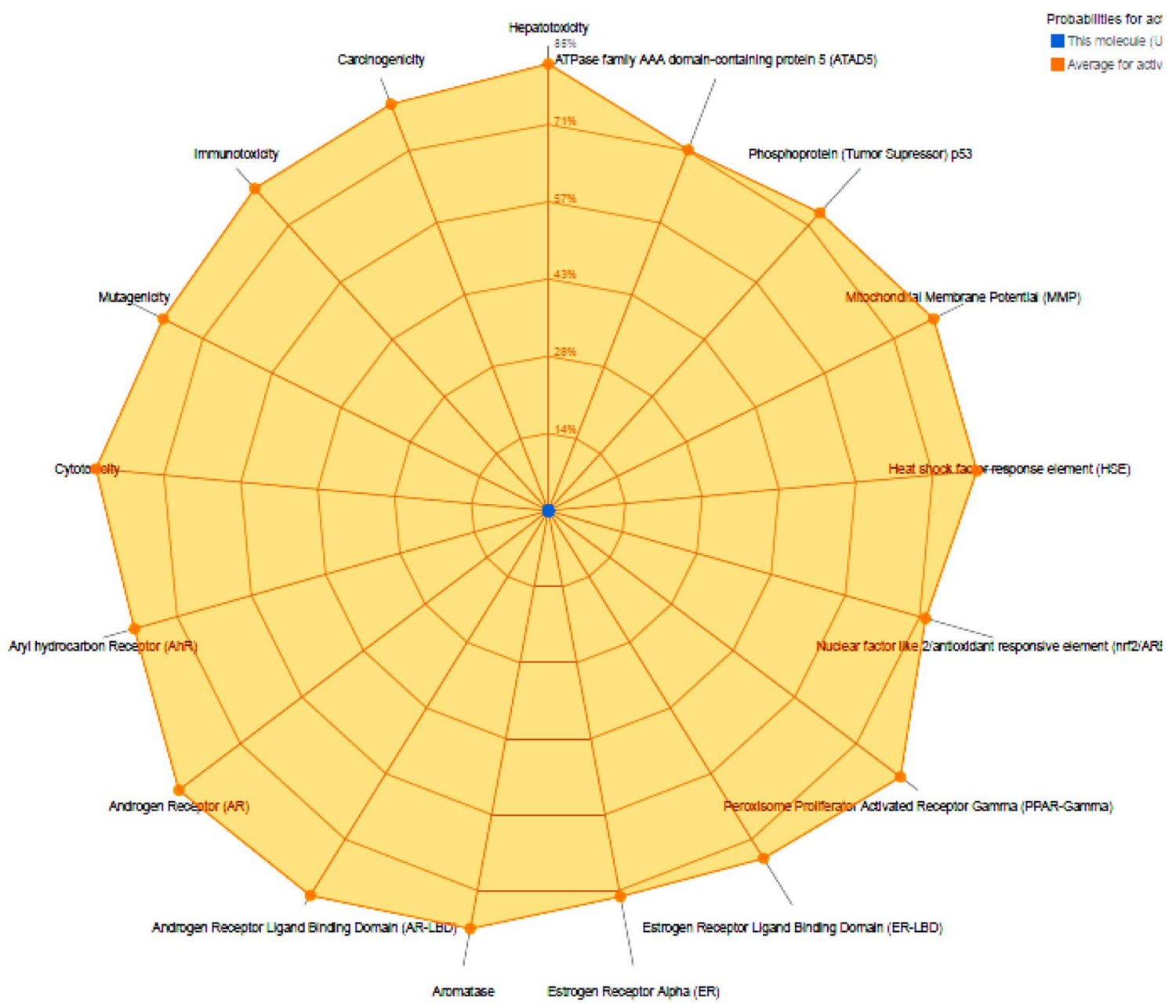

Fig. 4 Toxicity radar chart of 1,8 cineole

Table 6 Cancer cell line prediction result

\begin{tabular}{|c|c|c|c|c|c|}
\hline $\mathrm{Pa}$ & $\mathrm{Pi}$ & Cell line & Cell line Full name & Tissue & Tumor type \\
\hline 0.949 & 0.002 & $\underline{\text { NCI-H187 }}$ & $\begin{array}{c}\text { Small cell lung } \\
\text { carcinoma }\end{array}$ & Lung & Carcinoma \\
\hline 0.770 & 0.002 & $\underline{\text { Raji }}$ & $\begin{array}{c}\text { B-lymphoblastic } \\
\text { cells }\end{array}$ & $\begin{array}{c}\text { Haematopoietic and } \\
\text { lymphoid tissue }\end{array}$ & Leukemia \\
\hline 0.817 & 0.003 & $\underline{\text { BXPC-3 }}$ & $\begin{array}{c}\text { Pancreatic } \\
\text { adenocarcinoma }\end{array}$ & Pancreas & Adenocarcinoma \\
\hline 0.925 & 0.003 & $\underline{\text { LoVo }}$ & $\begin{array}{c}\text { Colon } \\
\text { adenocarcinoma }\end{array}$ & Colon & Adenocarcinoma \\
\hline 0.908 & 0.005 & $\underline{\text { A549 }}$ & Lung carcinoma & Lung & Carcinoma \\
\hline 0.701 & 0.005 & $\underline{\text { HCT-15 }}$ & $\begin{array}{c}\text { Colon } \\
\text { adenocarcinoma }\end{array}$ & Colon & Adenocarcinoma \\
\hline 0.750 & 0.005 & $\underline{\text { HepG2 }}$ & Hepatoblastoma & Liver & Hepatoblastoma \\
\hline 0.719 & 0.007 & $\underline{\text { PC-3 }}$ & Prostate carcinoma & Prostate & Carcinoma \\
\hline 0.511 & 0.011 & $\underline{\text { A2058 }}$ & Melanoma & Skin & Melanoma \\
\hline 0.605 & 0.032 & $\underline{\text { MCF7 }}$ & Breast carcinoma & Breast & Carcinoma \\
\hline
\end{tabular}

$P a$ (probability "to be active"), $P i$ (probability "to be inactive") 

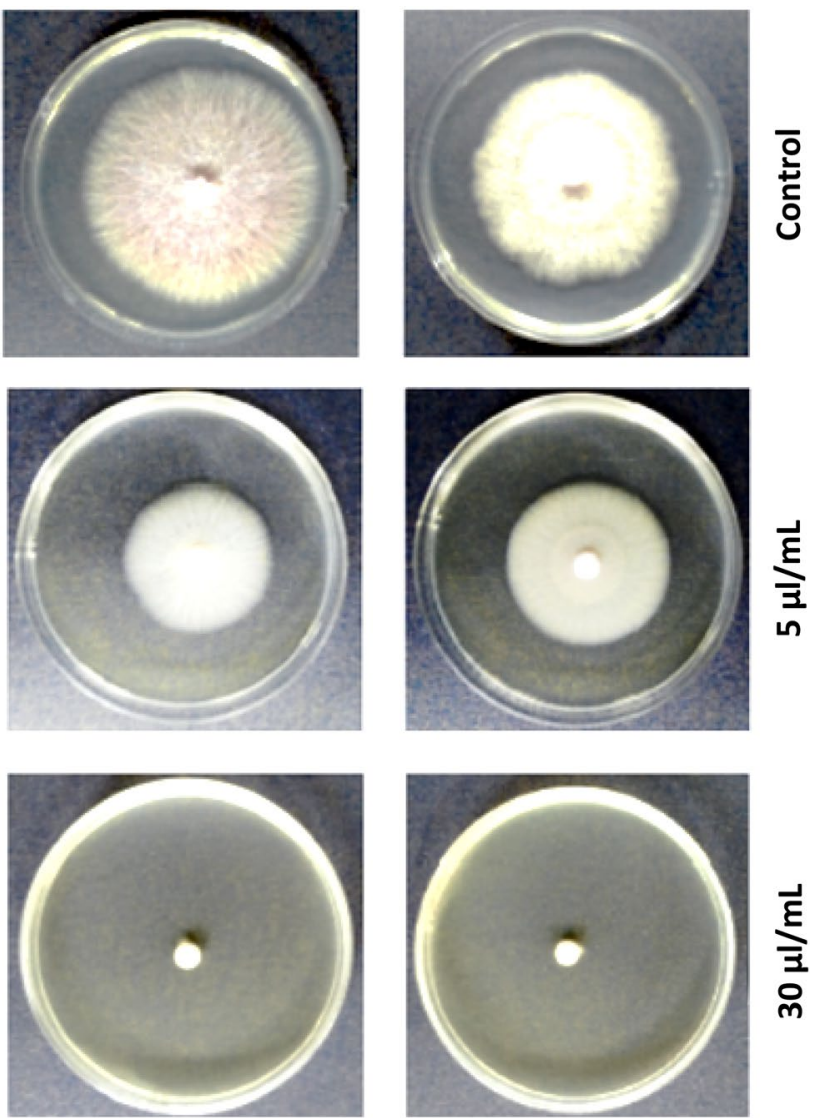

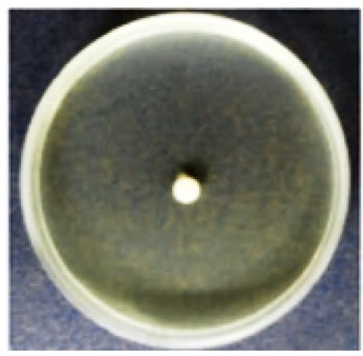

Aspergillus niger

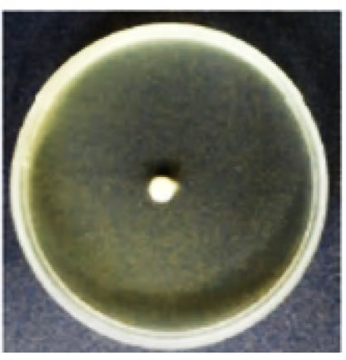

Aspergillus oryzae
Fig. 5 Effect of eucalyptus essential oil on fungal mycelial growth inhibition

Table 7 Mycelial growth inhibition rate (\%) of eucalyptus essential oil

Essential oil concentration ( $\mu \mathrm{L} / \quad$ Mycelial growth inhibition (\%) $\mathrm{mL}$ )

\begin{tabular}{ll}
\hline Aspergillus niger & $\begin{array}{l}\text { Asper- } \\
\text { gillus } \\
\text { oryzae }\end{array}$
\end{tabular}

\begin{tabular}{lll}
\hline 0 & Nil & Nil \\
5 & 50 & 40 \\
30 & 100 & 100 \\
Streptomycin & 100 & 100 \\
\hline
\end{tabular}

\section{Conclusion}

Aspergillosis has emerged as a pandemic in India. This study findings emanated from both in silico and in vitro revealed that eucalyptus essential oil due to the richness of eucalyptol or 1,8 cineole from eucalyptus essential oil plant could be promising antifungal therapeutic agents against chitin synthase, UDP-glycosyltransferase and Glucosamine-6-phosphate synthase protein.

Acknowledgements Authors want to acknowledge managing committee LKC

Funding Dept of Science and Technology, Govt. of India.

Availability of data and materials Not applicable.

\section{Declarations}

Conflict of interest The authors declares that they have no competing interest.

Ethics approval Not applicable.

Consent to participate Not applicable.

Consent for publication Yes.

\section{References}

1. Lai CC, Wang CY, Hsueh PR (2020) Co-infections among patients with COVID-19: the need for combination therapy with non-anti-SARS-CoV-2 agents? J Microbiol Immunol Infect 53:505-512

2. Alanio A, Dellière S, Fodil S, Bretagne S, Mégarbane B (2020) Prevalence of putative invasive pulmonary aspergillosis in critically ill patients with COVID-19. Lancet Respir Med 8:e48-e49

3. Koehler P, Cornely OA, Böttiger BW, Dusse F, Eichenauer DA, Fuchs F (2020) COVID-19 associated pulmonary aspergillosis. Mycoses 63:528-534

4. Chowdhary A, Tarai B, Singh A, Sharma A (2020) Multidrugresistant Candida auris infections in critically ill coronavirus disease patients, India, April-July. Emerg Infect Dis 26:2694-2696

5. Chang CC, Senining R, Kim J, Goyal R (2020) An acute pulmonary coccidioidomycosis coinfection in a patient presenting with multifocal pneumonia with COVID-19. J Investig Med High Impact Case Rep 8:2324709620972244

6. Poignon C, Blaize M, Vezinet C, Lampros A, Monsel A, Fekkar A (2020) Invasive pulmonary fusariosis in an immunocompetent critically ill patient with severe COVID-19. Clin Microbiol Infect 26:1582-1584

7. Werthman-Ehrenreich A (2020) Mucormycosis with orbital compartment syndrome in a patient with COVID-19. Am J Emerg Med S0735-6757(20):30826-30833

8. Ventoulis I, Sarmourli T, Amoiridou P, Mantzana P, Exindari M, Gioula G (2020) Bloodstream infection by Saccharomyces cerevisiae in two COVID-19 patients after receiving supplementation of Saccharomyces in the ICU. J Fungi (Basel) 6:98 
9. $\mathrm{Ku}$ YH, Chan KS, Yang CC, Tan CK, Chuang YC, Yu WL (2017) Higher mortality of severe influenza patients with probable aspergillosis than those with and without other coinfections. J Formos Med Assoc 116:660-670

10. John TM, Jacob CN, Kontoyiannis DP (2021) When uncontrolled diabetes mellitus and severe COVID-19 converge: the perfect storm for mucormycosis. J Fungi (Basel) 15:298

11. Rabagliati R, Rodríguez N, Núñez C, Huete A, Bravo S, Garcia $P$ (2021) COVID-19-associated mold infection in critically ill patients. Chile Emerg Infect Dis 27(5):1454-1456

12. Schweer KE, Bangard C, Hekmat K, Cornely OA (2014) Chronic pulmonary aspergillosis external icon. Mycoses 57:257-270

13. Cuenca-Estrella M (2014) Antifungal drug resistance mechanisms in pathogenic fungi: from bench to bedside. Clin Microbiol Infect 20:54-59

14. Chen X, Zewen Z, Zuozhong C, Yiman L, Shan S, Shujuan S (2020) Potential antifungal targets based on glucose metabolism pathways of Candida albicans. Front Microbiol 11:296

15. Han X, Zhu X, Hong Z, Wei L et al (2017) Structure-based rational design of novel inhibitors against fructose-1,6-bisphosphate aldolase from Candida albicans. J Chem Inf Model 57:1426-1438

16. Geoghegan I, Steinberg G, Gurr S (2017) The role of the fungal cell wall in the infection of plants. Trends Microbiol 25:957-967

17. Muniz M, Zurzolo C (2014) Sorting of GPI-anchored proteins from yeast to mammals-common pathways at different sites. J Cell Sci 127:2793-2801

18. Banerjee K, Gupta U, Gupta S, Wadhwa G, Gabrani R, Sharma SK, Jain CK (2011) Molecular docking of glucosamine-6-phosphate synthase in Rhizopus oryzae. Bioinformation 7:285-290

19. Sharma AD, Farmaha M, Kaur I, Singh N (2021) Phytochemical analysis using GC-FID, FPLC fingerprinting, antioxidant, antimicrobial, anti- inflammatory activities analysis of traditionally used eucalyptus globulus essential oil. Drug Analytical Res 5:26-38

20. Elaissi A, Bel Haj Salah K, Mabrouk S, Chemli R, Harzallah-Skhiri F (2011) Antibacterial activity and chemical composition of 20 Eucalyptus species essential oils. Food Chem 129:1427-1434

21. Sartorelli P, Marquioreto AD, Amaral-Baroli A, Lima ME, Moreno PR (2007) Chemical composition and antimicrobial activity of the essential oils from two species of Eucalyptus. Phytother Res 21:231-233

22. Singh P, Kumari K, Awasthi SK, Chandra R (2016) Virtual screening and docking studies of synthesised chalcones: potent anti-malarial drug. Int J Drug Dev Res 8:49-56

23. Barcellos MP, Santos CB, Federico LB, Almeida PF, da Silva CHTP, Taft CA (2019) Pharmacophore and structure-based drug design, molecular dynamics and admet/tox studies to design novel potential pad4 inhibitors. J Biomol Struct Dyn 37:966-981

24. Dorfmueller HC, Ferenbach AT, Borodkin VS, van Aalten DMF (2014) A structural and biochemical model of processive chitin synthesis. J Biol Chem 289:23020-23028

25. Mouilleron S, Badet-Denisot MA, Golinelli-Pimpaneau B (2008) Ordering of C-terminal loop and glutaminase domains of glucosamine-6-phosphate synthase promotes sugar ring opening and formation of the ammonia channel. J Mol Biol 377(4):1174-1185

26. Wojciechowski M, Milewski S, Mazerski J, Borowski E (2005) Glucosamine-6-phosphate synthase, a novel target for antifungal agents. Molecular modelling studies in drug design. Acta Biochim Pol 52:647-653

27. Sawitri WD, Afidah SN, Nakagawa A, Hase T, Sugiharto B (2018) Identification of UDP-glucose binding site in glycosyltransferase domain of sucrose phosphate synthase from sugarcane (Saccharum officinarum) by structure-based site-directed mutagenesis. Biophys Rev 10(2):293-298

28. Omar HS, Abd El-Rahman SN, AlGhannam SM, Sedeek MS (2021) Antifungal evaluation and molecular docking studies of
Olea europaea leaf extract, Thymus vulgaris and Boswellia carteri essential oil as prospective fungal inhibitor candidates. Molecules 26:6118. https://doi.org/10.3390/molecules26206118

29. Biswal AR, Venkataraghavan R, Pazhamalai V, Romauld S (2019) Molecular docking of various bioactive compounds from essential oil of Trachyaspermum ammi against the fungal enzyme Candidapepsin-1. J Appl Pharm Sci 9:21-32. https://doi.org/10.7324/ JAPS.2019.90503

30. Kortemme T, Morozov AV, Baker D (2003) An orientationdependent hydrogen bonding potential improves prediction of specificity and structure for proteins and protein-protein complexes. J Mol Biol 326:1239-1259

31. Lima SL, Colombo AL, de Almeida Junior JN (2019) Fungal cell wall: emerging antifungals and drug resistance. Front Microbiol 10:2573

32. Wu C, Liu Y, Yang Y, Zhang P, Zhong W, Wang Y, Wang Q et al (2020) Analysis of therapeutic targets for SARS-CoV-2 and discovery of potential drugs by computational methods. Acta Pharm Sin B 10:766-788

33. Konig J, Muller F (2013) Transporters and drug-drug interactions: Important determinants of drug disposition and effects. Pharmacol Rev 65:944-966

34. Srimai V, Ramesh M, Parameshwar KS, Parthasarathy T (2013) Computer-aided design of selective Cytochrome P450 inhibitors and docking studies of alkyl resorcinol derivatives. Med Chem Res 22:5314-5323

35. Abraham DJ (2003) Burger's medicinal chemistry and drug discovery volume 5 : chemotherapeutic agents. Wiley

36. Khan T, Dixit S, Ahmad R, Raza S, Azad I, Joshi S, Khan AR (2017) Molecular docking, PASS analysis, bioactivity score prediction, synthesis, characterization and biological activity evaluation of a functionalized 2-butanone thiosemicarbazone ligand and its complexes. J Chem Biol 10:91-104

37. Dar AM, Khan MA, Mir S, Gatoo MA (2016) DNA binding, cleavage activity, molecular docking, cytotoxicity and genotoxicity studies of newly synthesized copper based metal complexes. Pharm Anal Acta 7:464

38. Banerjee P, Eckert AO, Schrey AK, Preissner R (2018) ProTox-II: a webserver for the prediction of toxicity of chemicals. Nucleic Acids Res 46(W1):W257-W263

39. Raies AB, Bajic VB (2016) In silico toxicology: computational methods for the prediction of chemical toxicity. Wiley Interdiscip Rev Comput Mol Sci 6:147-172

40. Siramshetty VB, Nickel J, Omieczynski C, Gohlke BO, Drwal MN, Preissner R (2016) WITHDRAWN-a resource for withdrawn and discontinued drugs. Nucleic Acids Res 44:D1080-D1086

41. Lea IA, Gong H, Paleja A, Rashid A, Fostel J (2017) CEBS: a comprehensive annotated database of toxicological data. Nucleic Acids Res 45:D964-D971

42. Huang R, Xia M, Sakamuru S, Zhao J, Shahane SA, AtteneRamos M, Zhao T, Austin CP, Simeonov A (2016) Modelling the Tox $2110 \mathrm{~K}$ chemical profiles for in vivo toxicity prediction and mechanism characterization. Nat Commun 7:1-10

43. Rooke D, Shattock R (1983) Chloramphenicol and Streptomycin on Developmental Stages of Phytophthora infestans. J Gen Microbiol 129:3401-3410

44. Singh G, Gupta S, Sharma N (2014) In vitro screening of selected plant extracts against Alternaria alternate. J Expt Biol 2:344-351

45. Katooli N, Maghsodlo R, Razavi SE (2011) Evaluation of eucalyptus essential oil against some plant pathogenic fungi. J Plant Breed Crop Sci 3:41-43

46. Mehani M, Salhi N, Valeria T, Ladjel S (2014) Antifungal effects of essential oil of Eucalyptus camaldulensis plant on Fusarium graminearum and Fusarium sporotrichioides". Int J Current Res 6:10795-10797 